the Western Electric Co. conducted a great experiment from the American naval station at Arlington, near Washington, and succeeded in speaking to the Eiffel Tower (3700 km.), and exceptionally to. Honolulu $(8000 \mathrm{~km}$.). Triodes were used as oscillators, modulators, and magnifiers. The chain of apparatus was as follows: First came a triode oscillator of small power, which was coupled by means of a highfrequency transformer to the grid circuit of a medium-sized tube. This grid circuit contained also the secondary winding of a transformer, the primary of which carried the currents from the microphone. The anode circuit of this medium triode therefore contained magnified modulated current of the high frequency dictated by

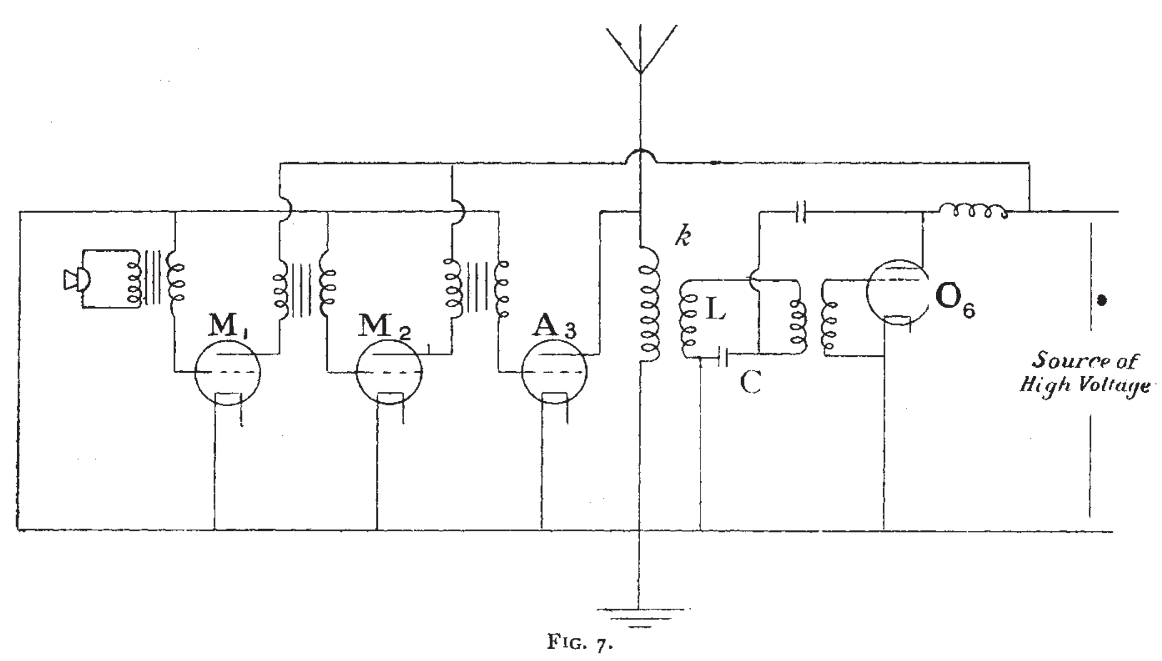

type. A diagram of the chief parts appears in Fig. 7, from which are omitted all details regarding the heating currents for the filaments and concerning the rectufying of the high-voltage current for the anode circuits. The oscillations are generated in the circuit $\mathrm{LC}$ by a bank of six large three-electrode valves in parallel marked $\mathrm{O}_{0}$, and transferred to the antenna by the coupling $k$. The absorption tubes are three in number, and are shown at $\mathrm{A}_{3}$; their three anodes are large enough to dissipate all the energy normally given to the antenna. These absorption triodes are controlled by applying to their grids the speech electromotive forces after these have been magnified by the successive tricdes $M_{1}$ and $M_{2}$. The total consumption of power is $20 \mathrm{kw}$., including all that necessary for heating the filaments, the height of the aerial is $400 \mathrm{ft}$., and the wavelength $275^{\circ}$ metres.

It will be noticed that the above are all oneway methods-that is to say, the two persons using two stations for conversation must speak in turn, and the listener must wait for the speaker to finish before he switches over from his listening circuit to his speaking circuit. This falls far short of perfection. For perfect telephony it is essential that both persons shall

the small triode oscillator. The current was next transferred by means of a transformer from this anode circuit to the grid circuit of a bank of medium-sized amplifying tubes connected in parallel, and was again magnified, and finally it was transformed once more into a circuit containing the grids of more than 500 parallel connected tubes. It ought to be remarked that great progress has been made since 1914 in the manufacture of power bulbs, and that the experiment can now be carried out on a larger power scale with a far smaller array of tubes.

The remaining example to be cited is the recently erected $20-\mathrm{kw}$. plant of the Marconi Co. During the past few weeks it has transmitted good quality speech to a distance of 1500 miles. In principle it is of the subtraction or absorption be able to speak and hear simultaneously if they so desire, as in ordinary line telephony. For information about the most modern attempts at duplex telephony a paper read recently before the Institution of Electrical Engineers by Mr. P. P. Eckersley should be consulted. Until a complete duplex method is devised and proved, wireless telephony must remain a somewhat irksome mode of communication for public use. The ideal method will be such that a wireless station on this side of the Atlantic could be connected over land lines to, say, London, and a station on the other side connected by land lines to New York, and the persons using the apparatus would be unable to tell that wireless telephony across the ocean had been an agency in the transmission of their voices.

\title{
The Meteorology of the Temperate Zone and the General Atmospheric Circulation.
}

\section{By Prof. V. Bjerknes.}

IN Norway, since the year I9I8, an attempt has been made to base forecasts of weather on the application of a very close network of meteorological stations. The study of the corresponding very detailed synoptic charts has led to interesting results even for large-scale meteorNO. 2643 , VOL. IO5] ology. These are due especially to three young meteorologists, J. Bjerknes, H. Solberg, and T. Bergeron, who have been attached to this service, and will return to the subject in detailed papers.

A very short summary of some of the main 
results will be given here. These will be seen to give, to some extent, both verifications and further developments of ideas, which, although advanced by leading theoretical meteorologists, have not yet exerted any noticeable influence upon the development of meteorology. ${ }^{1}$

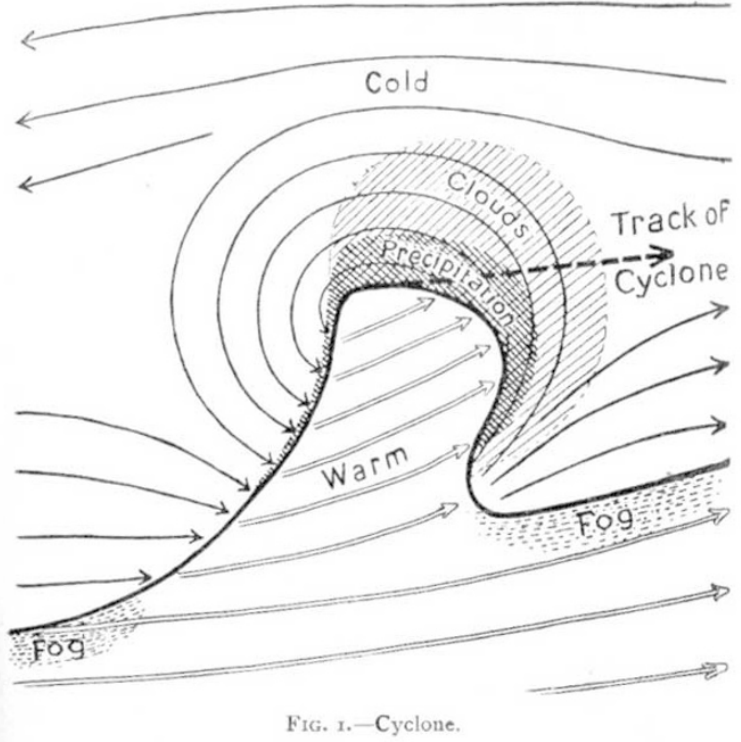

Great changes in the weather in our latitudes have been found to depend upon the passage of a line of discontinuity which marks the frontier between masses of air of different origin. A line of this kind was first found to exist in every current of cold air (Fig. I). The whole system moves with the east-bound current, and the cyclonic centre with the lowest pressure is in the region where the cyclonic track touches the border of the tongue. The front border, before this point, is curved like a reversed " $S$ "; the rear border, behind this point, has a uniform concave curvature. Along the front border warm air from the tongue ascends the barrier formed by the cold air, which, in return, passes round the tongue in order to penetrate below the warm air along the rear border. Two bands of rain are thus formed -a broad one in front of the tongue, where the warm air spontaneously surmounts the cold, and a narrow one, generally called the squall line, along the rear border, where the advancing cold air violently lifts the warm air of the tongue. ${ }^{2}$

It has been found by use of the detailed maps that the line of discontinuity exists even outside the cyclone, passing from one cyclone to the other; they follow each other along a common line of discontinuity, like pearls on a string.

When one has become acquainted with all the signs-direct and indirect-which are seen to indicate the position of a line of discontinuity on the very detailed maps, it proves possible to discover them even on less detailed maps. Fig. 2 shows roughly the course of such a line, on January I, 1907, as it may be drawn upon the Hoffmeyer maps of the Atlantic Ocean for that day. When similar charts are drawn from day to day, as accurately as circumstances allow, a series of large-scale results very distinctly presents itself.

Though we have been able to draw the line only half round the pole, there can be no doubt that

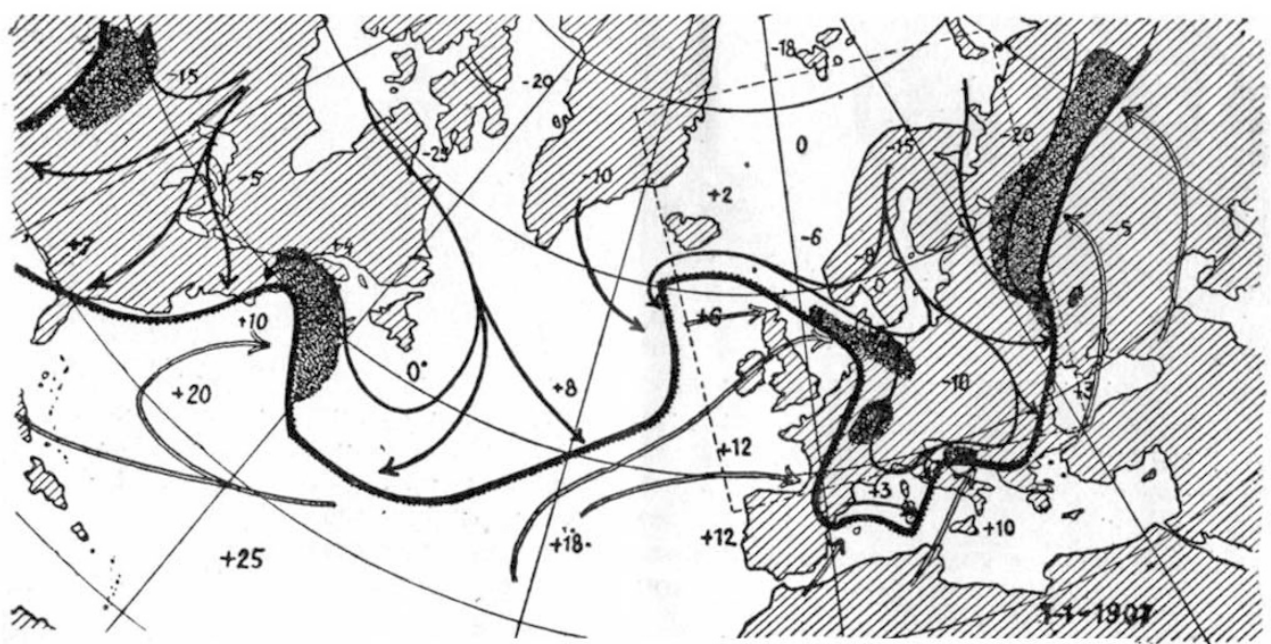

FIG. 2,-Line of demarcation between polar and equatorial air, January I, I907

cyclone which is not perfectly stationary. It here borders a tongue of warm air, which from an east-bound current penetrates into a west-bound 1 Dove: "Das Gesetz der Stürne," Vierte Auflage (Berlin, 1873). Helmholtz: "Ueber atmosphärische Bewegungen," Sitzungsberichte der K. Preuss. Akad. der Wissenschaften r888, Meteorolngische Zeitschrift, 1888. Brillouin: "Vents Contigus et Nuages," Annales du Bureau Cen'ral Mttéorologique, 1898 . M argules: "Energie der Stürme," Jahrbuch der K. K. Centralanstalt für Meteorologie, 1903, Anhang.

NO. 2643, VOL. IO5] it surrounds the polar regions as a closed circuit. On the northern side of this line all signs indicate air of polar origin; it has a low temperature for the latitude, shows great dryness, distinguishes itself by great visibility, and has a prevailing

2 Cf, W. N. Shaw: "Forecasting Weather," p. 212 (London, rgrz) J. Bjerknes: "On the Structure of Moving Cyclones," Geofysiske Publikationer (Kristiania, I919). 
motion from east and north. On the southern side of the line the tropical origin of the air is recognised by the corresponding signs-its generally higher temperature, its greater humidity, its haziness, and its prevailing motion from west and south. There can then be no doubt concerning the origin of the line. Heavy, cold air flows out along the ground from the polar regions. It is separated from the overlying warmer air by a surface of discontinuity, the height of which above the ground decreases very slowly until it cuts the ground along our line of discontinuity. Thus this line shows how far the cold air has succeeded in penetrating; it is a kind of polar front line.

Along the whole of this front line we have the conditions, especially the contrasts, from which atmospheric events originate-the strongest winds, the most violent shifts of wind, and the greatest contrasts in temperature and humidity. Along the whole of the line formation of tog, clouds and precipitation is going on, fog prevailing where the line is stationary, clouds and precipitation where it is moving.

The line has a wavy form, and is in a continuous undulating motion, thereby sweeping over the whole of what is called the temperate zone. The wavy form comes from alternately cold and warm tongues of air, which extend themselves towards the equator or the pole. The whole system is moving from west to east, while the line, at the same time, changes its form, especially when great masses of accumulated cold air are expelled from the central polar regions. The more wavy the form of the line, the more tempestuous and variable is the weather. At the northern ends of the warm tongues the air motion which characterises cyclones is recognised, and the corresponding areas of rain are seen so far as it has been possible to mark them from the few observations; these are the places of great storms and low barometric pressure. The broad tongues of polar air, on the other hand, bring the clearing up between the successive storms and the corresponding higher barometric pressure.

Two expanding tongues of cold air may occasionally cut off from its base an interjacent tongue of warm air. Then the storm at the polar end is no longer supplied by warm air, and soon loses its power; this is the death of a cyclone. A tongue of polar air which has extended itself too much towards the tropics may be cut off in a similar way; or, as the consequence of a new outbreak of polar air, a more retired front may be formed behind one too far advanced. In this way great isolated isles of polar air are formed in lower latitudes; this gives the formation of great anticyclones, which generally bring settled, good weather. Thus anticyclones are born as cyclones die. Cyclone and anticyclone and all meteorological events of the temperate zone are in the most intimate way related to the polar front and its motion.

This expulsion of great masses of polar air, which leads to the formation of anticyclones, also enters as an essential element into the great atmospheric circulation. There is a practically continuous flow of warm air along the ground from the "highs" of the sub-tropic calms towards the polar regions. This flow concentrates itself in the warm tongues, and continues into the polar regions in upper levels. Here the air is cooled, and eventually reaches lower levels. Thus increasing masses of cooled air are accumulated behind the polar front. This must continuously advance, with the effect that the tracks of the corresponding cyclones are always moved further towards the south. Finally, at the place of least resistance, great masses of cold air break through and are expelled in the direction of the tropics. The polar front performs a corresponding retreat, the cyclonic tracks are again displaced to the north, and the type of weather is changed. Then the same action repeats itself. This intermittent form of the great atmospheric circulation is especially pronounced in the winter. During the summer the polar front is far back, and the high temperature of the continents exerts a considerable influence; then occasionally a continuous return of polar air may be established along the west coast of the continents, leading direct into the trade winds.

These results cannot fail to exert a considerable influence upon the methods of weather forecasting. All meteorological events of the temperate zone, great and small, are derived from the general atmospheric circulation described above, as we know it from the motions of the polar front. If we succeed in watching it effectively it should be possible not only to give short-range forecasts a hitherto unattained accuracy, but also to complete them by long-range forecasts giving the general character of the weather perhaps for weeks ahead. These two kinds of forecast could be extended to all regions of the temperate zone -oceanic as well as continental. The required survey of the polar front is merely a question of organisation.

\section{The Cardiff Meeting of the British Association.}

PREPARATIONS are going steadily forward in Cardiff for the forthcoming meeting of the British Association from Tuesday; August 24, to Saturday, August 28 inclusive. Owing to the crowded state of the town, the question of accommodation is causing difficulties, but these will, it is expected, be satisfactorily overcome. A list of NO. $2 \overline{6} 43$, VOL. IO5] hotels and lodgings will be issued at the end of the present week.

The Marquis of Bute has generously offered to give a garden party at the Castle on the Wednesday -afternoon, and the invitation has been gratefully accepted by the Lord Mayor on behalf of the local executive committee. Owing to the 